\title{
Editorial
}

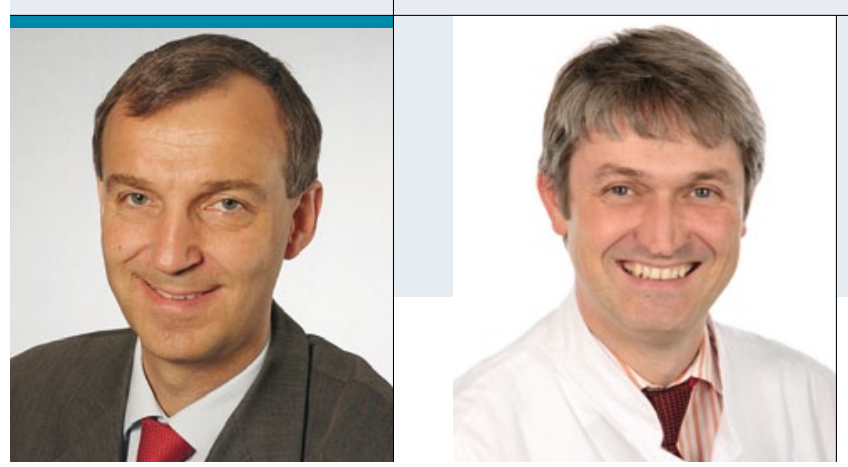

„Wir wollen eine Brückeschlagen zwischen der Physiologie der Haut und der Rolle der Immunologie bei atopischer Dermatitis."

Prof. Dr. Thomas Werfel, Klinik für Dermatologie, Allergologie und Venerologie, Med. Hochschule Hannover Prof. Dr. Tilo Biedermann, Universitäts-Hautklinik, EberhardKarls-Universität, Tübingen

\section{Atopische Dermatitis - neue Blickwinkel, neue Therapien}

$\mathrm{V}$ oraussetzung für neue Therapieansätze ist das Verständnis von Krankheitsprozessen. Nicht selten aber haben wir durch die Erforschung einer Krankheit auch physiologische Prozesse erst richtig verstanden. Dies trifft auch auf die atopische Dermatitis zu. Unsere Haut hat sich - bedingt durch den stetigen Kontakt mit der Umwelt - im Laufe der Evolution in besonderem Maße spezialisieren müssen. Aufregende Forschungsergebnisse der letzten Jahre haben eine Reihe von Funktionen der Haut und ihrer Rolle als Oberflächenorgan entschlüsselt. Durch die Identifikation der Rolle der Barriere für die atopische Dermatitis haben wir z.B. gelernt, welche Bedeutung Moleküle wie das Filaggrin für unsere Haut haben.

Aufgeteilt auf zwei Ausgaben des Allergo Journal werden wir Ihnen ein echtes Update zur atopischen Dermatitis präsentieren. In diesem Heft werden in einer CME-zertifizierten Übersichtsarbeit neueste Erkenntnisse rund um die Therapie des atopischen Ekzems dargestellt (S. 404). Dabei wird Bewährtes und neu Bewertetes nebeneinander gestellt und ein Ausblick auf neue Entwicklungen gegeben. Diese Aufstellung beschreibt die (best-)möglichen Therapieansätze für unsere Patienten mit atopischer Dermatitis - die Wirklichkeit sieht aber leider oft ganz anders aus. Deshalb haben wir diesem Artikel eine Studie zur Versorgung von Patienten mit atopischem Ekzem zur Seite gestellt (S. 418). Derartige Studien werden helfen die Versorgungssituation der Patienten zu verbessern. Um die Bedeutung des Immunsystems und der Immunregulation für die atopische Dermatitis geht es dann im Allergo Journal 8/2012.

Die Charakterisierung des Immunsystems und seine Einteilung in das natürliche bzw. angeborene Immunsystem sowie das adaptive Immunsystem sind ein entscheidender Schritt zum besseren Verständnis von Krankheitsprozessen der Haut. Wir wissen heute, dass Immunfunktionen Teil der Homöostase der Haut sind und ihre Barrierefunktion regulieren, das Mikrobiom bestimmen sowie Abwehrfunktionen einleiten, richtig dosieren und auch terminieren. Wenn wir lernen, wie das Mikrobiom ohne Entzündung vertragen wird, können wir dieses Konzept auch therapeutisch einsetzen. Im nächsten Allergo Journal wird deshalb näher ausgeführt, wer die wichtigen Spieler in diesem Immun-Team sind und wie sie in entzündeter Haut bei Patienten mit atopischer Dermatitis im Vergleich zu Hautgesunden verändert sind und wie Fehlregulationen des Immunsystems zur häufigen Kolonisierung der Haut bei atopischer Dermatitis mit Staphylokokken, Herpes- oder Molluskenviren beitragen.

Der atopischen Dermatitis widmet sich das Allergo Journal aus dem Grund so differenziert, da auch Allergien Ausdruck einer hochspezialisierten Immunregulation der Haut sein können. $\mathrm{Zu}$ nennen sind hier z. B. Nahrungsmittelallergien, deren Abklärung insbesondere in der Kindheit bei atopischer Dermatitis einen hohen Stellenwert hat. Auch mikrobielle Antigene auf der Haut können bei Atopikern zu Allergenen „mutieren“, wenn diese eine Th2-Antwort induzieren. Autoantigene führen bei atopischer Dermatitis eventuell sogar zur „Autoallergie“

Neue Blickwinkel auf die Entstehung der atopischen Dermatitis eröffnen auch neue therapeutische Möglichkeiten! Wir wollen Sie gerne auf diese spannende Reise mitnehmen, indem wir eine Brücke schlagen zwischen der Physiologie des Oberflächenorgans Haut, der Rolle der Immunologie und Allergie bei atopischer Dermatitis und der Perspektive „Translation" von hochaktuellen Themen aus der Forschung in die Klinik von heute.

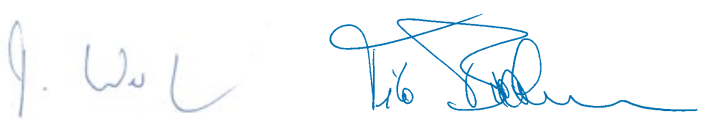

